\title{
Pseudorandom Dynamic Test Power Signal Modeling and Electrical Energy Compressive Measurement Algorithm
}

\author{
Xuewei Wang, Jing Wang \\ Institute of Information Science and Technology, Beijing University of Chemical Technology, North Third Ring Road, No.15, \\ 100029, Beijing, China,wangxw@mail.buct.edu.cn
}

\begin{abstract}
With the rapid construction of smart grid, many applications of the new generation and the large power dynamic loads are revolutionizing the electrical energy measurement of electricity meters. The dynamic measurement errors produced by electricity meters are intolerable. In order to solve the dynamic error measurement of electrical energy, firstly, this paper proposes a three-phase pseudorandom dynamic test power signal model to reflect the main characteristics of dynamic loads. Secondly, a compressive measurement algorithm is proposed by the means of steady-state optimization to accurately measure the electrical energy. The experimental results confirm the effectiveness of the three-phase pseudorandom dynamic test signal model, the maximum errors of compressive measurement algorithm are superior to $1 \times 10^{-13}$, the high precision enables the algorithm to accurately measure the electrical energy under different dynamic conditions.
\end{abstract}

Keywords: Compressive measurement, dynamic load, electricity meter, electrical energy.

\section{INTRODUCTION}

During recent years, the electrical networks have been growing more and more complex, with the applications of distributed renewable electricity sources (such as photovoltaic power supply) and the use of dynamic loads (such as electric arc furnaces, high-speed railway train and electric vehicle charging station), the current amplitude fluctuations of the dynamic loads have shown complex random characteristics, such as the quick and random dynamic changes [1]-[4]. Under these dynamic conditions, the electrical energy measured by electricity meters may produce significant measurement errors, in some cases, the error can be as large as $-37.7 \%$ [5]. Therefore, in order to evaluate electrical energy measuring algorithm under dynamic conditions, dynamic test signal modeling and the investigation of electrical energy measuring algorithm have become two challenging scientific problems.

The test signal modeling is important for reflecting the influence of dynamic load characteristics to electrical energy measuring algorithms. Last decades have witnessed significant contributions to this topic; they are essentially focused on the steady periodic test signal modeling, including sinusoidal and nonsinusoidal signals [6]-[9]. The standard IEEE 1459 clearly has defined the steady nonsinusoidal signal model with harmonics [10], [11]. In recent years, a few studies have started focusing on the dynamic test signal modeling. The authors in [12] and [13] have proposed two special amplitude modulation test signals with sine wave envelope or trapezoidal wave envelope to reflect the influence of current amplitude periodic fluctuation to the measuring algorithm in electricity meter. In [5], the on-off-keying (OOK) dynamic test power signal model has been proposed for evaluating the influence of current amplitude quickly changing to electrical energy measuring algorithm. The above proposed signal models are effective for solving the error evaluation of electrical energy measuring algorithm adopted in electricity meters under steady or periodical dynamic conditions. However, in electrical networks, the dynamic load current amplitude changes with random characteristics, obviously, the major drawbacks of these signal models are that they are deterministic and periodical, that is to say, they cannot reflect complex random dynamic test characteristics. Unfortunately, until now no suitable dynamic test signal models can be found in both literatures and standards.

As for the electrical energy measuring algorithm, the conventional algorithms are in time domain or frequency domain. In the time domain, the electrical energy measuring algorithms, including asynchronous measuring algorithm [14], quasi-synchronous measuring algorithm [15] and in the frequency domain, the widely used electrical energy measuring algorithms, are based on cosine window discrete Fourier transform (DFT) algorithms [16], [17]. However, until now, the errors of the above energy measuring algorithms have not been clear under dynamic random conditions. 
As for the random dynamic signal processes, the compressive sensing (CS) is a very efficient signal processing theory [18], [19], which states that if the observed signal is sparse in some transform domains, according to the restricted isometry property (RIP), it is possible to reconstruct the signal with high probability from many fewer samples by constructing sensing matrices. Up to now, the CS signal processing has been investigated in different fields and has mainly focused on signal compression, reconstruction, and sensing matrix construction [20]-[23].

In CS, the sensing matrix construction is an important part for signal measurement. The proposed sensing matrices can be divided into three main classes: random matrices [24][26], deterministic matrices [27]-[29], and optimal sensing matrices for particular signal processing problems, such as the matrix with enhanced ratio of signal-to-interference [30] and the matrix with the minimal relevance to sparse bases [31].

In order to solve signal detection problems and to avoid the high computational complexity of signal reconstruction, there is a growing interest to investigate a novel signal processing method that directly deals with compressive measurements, which is called the CM method. In [32], a novel CM algorithm has been proposed based on the Gaussian matrix for signal detection, classification and estimation. After that, the authors in [23] have investigated a $\mathrm{CM}$ algorithm for detecting machine fault features by using the Walsh-Hadamard sensing matrix. The authors in [33] have proposed a $\mathrm{CM}$ algorithm for state detection of vibration signal. However, the existing $\mathrm{CM}$ algorithms have mainly focused on the detection or estimation of random signal feature and state. But little attention has been paid to the algorithm for accurately measuring electrical energy value under random dynamic conditions.

In this paper, we have the motivations to estimate a novel pseudorandom dynamic test signal model for evaluating errors of the cosine window-based electrical energy measuring algorithms and investigate a novel CM algorithm for accurately measuring the electrical energy value under random dynamic conditions. The main contributions in this paper are as follows:

- A three-phase pseudorandom dynamic test signal model is proposed to reflect the main random characteristics of dynamic loads.

- A keying implementation scheme is established to generate dynamic test current and power.

- A novel non-reconstructed CM algorithm is proposed for accurate electrical energy measurement.

- An optimal sensing matrix with minimum error is constructed for CM algorithm.

The rest of this paper is organized as follows: In Section 2, three-phase pseudorandom dynamic test current and power signals are proposed and generated. In Section 3, the sparseness of dynamic test power is proved. In Section 4, the $\mathrm{CM}$ algorithm is proposed for measuring electrical energy and an optimal sensing matrix is constructed. In Section 5, simulations are carried out to prove the accuracy of the CM algorithm and a discussion is also presented. Finally, Section 6 concludes this paper.

\section{PSEUDORANDOM DYNAMIC TEST SIGNAL MODELING AND IMPLEMENTATION SCHEME}

\section{A. Main characteristics of dynamic loads}

Under dynamic load conditions, the voltage is basically stable and periodical [34]. As far as the current and power signals are concerned, the main characteristics are as follows:

1) Amplitude random fluctuation: This characteristic is caused by the load operations [35], as shown in Fig.1. In this paper, a typical dynamic load of high-speed railway train (HSRT) is taken as an example.

2) Quasi-periodical fluctuation: As for the majority of dynamic loads, the current amplitudes fluctuate quasiperiodically (hour to hour, day to day) due to the operation period of load equipment [36], [37].

3) Approximate Gaussian distribution [38], [39]: Through analyzing the statistic distributions of dynamic loads, the current and power probability density functions of HSRT are shown in Fig.2. Obviously, the probability density functions are similar to the Gaussian distribution.
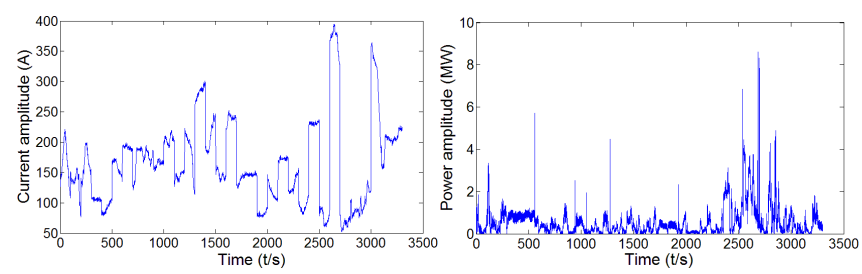

Fig.1. Current and power amplitude fluctuations of HSRT.

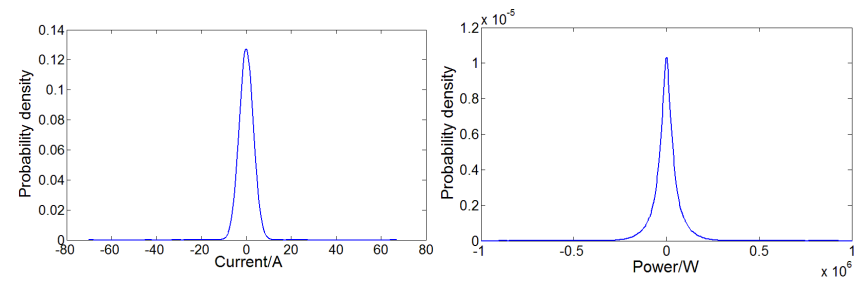

Fig.2. Current and power probability density functions of HSRT.

\section{B. Dynamic test signal modeling}

$\mathrm{m}$-Sequence forms a family of binary pseudorandom sequences with excellent correlation property, approximate Gaussian distribution characteristics, amplitude random change and quasi-periodical fluctuation [40]. It is worth noting that the m-sequence has similar characteristics as the current and power signals of dynamic loads, therefore, the $\mathrm{m}$-sequence is suitable for generating dynamic test signals. The voltage signal is kept in sinusoidal, a three-phase msequence dynamic test current and power signals are deduced, which can be seen as three-phase m-sequence Amplitude-Shift-Keying (ASK) signals.

The three-phase steady test voltage signal and dynamic test current signal can be expressed as:

$$
\begin{gathered}
u_{k}^{s}(t)=U_{k} \sin \left(\Omega_{1} t+\varphi_{k}\right) \\
i_{k}^{d}(t)=m(t) i_{k}^{s}(t)=I_{k} m(t) \sin \left(\Omega_{1} t+\phi_{k}\right)
\end{gathered}
$$


where $k=a, b, c$ represents the A-phase, B-phase, and Cphase, respectively. $u_{k}^{s}(t)$ and $i_{k}^{s}(t)$ are the steady voltage and current signal, respectively, $i_{k}^{d}(t)$ is the dynamic test current signal, $U_{k}$ is the amplitude of voltage, $I_{k} m(t)$ is the amplitude of dynamic current, $\varphi_{k}$ and $\phi_{k}$ are the initial phases of steady voltage and dynamic current, respectively. $f_{1}$ is the fundamental frequency and $f_{1}=50 \mathrm{~Hz} . \Omega_{1}$ is the angular frequency and $\Omega_{1}=2 \pi f_{1}, m(t)$ is the m-sequence binary modulation signal, which is:

$$
\begin{gathered}
m(t)=\sum_{r=1}^{N} m(r) g(t-r T)=\sum_{n}^{N}\left\{\sum_{z=1}^{n^{\prime}} C_{z} m(r-z)(\bmod 2)\right\} g(t-r T) \\
m(r)=\sum_{z=1}^{n^{\prime}} C_{z} m(r-z)(\bmod 2) r \in[N]:\left\{\left\{\cdots 2^{n^{\prime}}-1\right\}\right. \\
g(t-r T)=\left\{\begin{array}{l}
1, t \in[r T,(r+1) T] \\
0, t \notin[r T,(r+1) T]
\end{array}\right.
\end{gathered}
$$

where (4) is the m-sequence recursive formula, $T$ is the fundamental period and $T=1 / f_{1}, n^{\prime}$ is the level of $\mathrm{m}$ sequence, $N$ is the maximum length in a period of $\mathrm{m}$ sequence and $N=2^{n^{\prime}}-1, T_{m}$ is the period time of $\mathrm{m}$ sequence and $T_{m}=T N, r$ is an integer number. $C_{z}$ is 1 or 0 .

Based on the above analysis, the modulation model of the dynamic test current signal is shown in Fig.3.

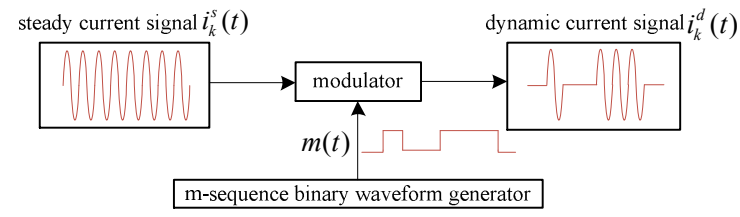

Fig.3. Modulation model of dynamic test current signal.

According to circuit theory, the mathematical expression for the calculation of the dynamic test power signal $p_{k}^{d}(t)$ is:

$$
\begin{aligned}
p_{k}^{d}(t) & =i_{k}^{d}(t) \cdot u_{k}^{s}(t)=m(t) \cdot p_{k}^{s}(t) \\
& =\frac{I_{k} U_{k}}{2} m(t)\left[\cos \left(\varphi_{k}-\phi_{k}\right)-\cos \left(2 \Omega_{1} t+\varphi_{k}+\phi_{k}\right)\right]
\end{aligned}
$$

where $p_{k}^{s}(t)=i_{k}^{s}(t) \cdot u_{k}^{s}(t)$, which is the steady test power.

\section{Implementation scheme of dynamic test power signal}

According to (1), (2), and (6), a keying implementation scheme is established to generate the $\mathrm{m}$-sequence dynamic test current and power as shown in Fig.4.

Based on the scheme, in actual experiments, using Fluke $6100 \mathrm{~A}$ as the steady power source to generate the steady voltage and current, the $\mathrm{m}$-sequence is used to control a silicon-controlled rectifier switch to ON or OFF to generate dynamic test current, as shown in Fig.5. Meanwhile, the dynamic test power is given from the multiplication of voltage $u_{k}^{s}(t)$ and current $i_{k}^{d}(t)$, as shown in Fig.6.

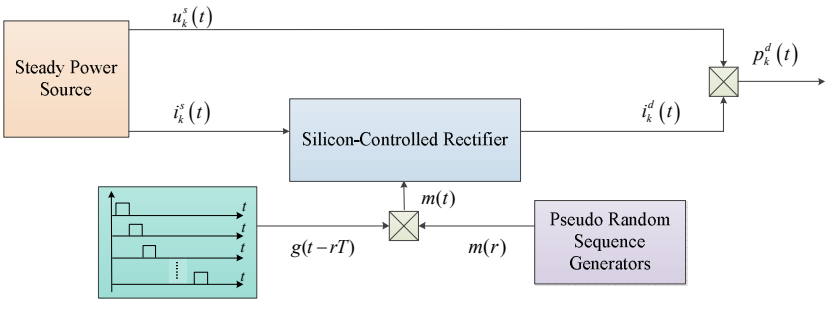

Fig.4. Keying implementation scheme of m-sequence dynamic test current and power.

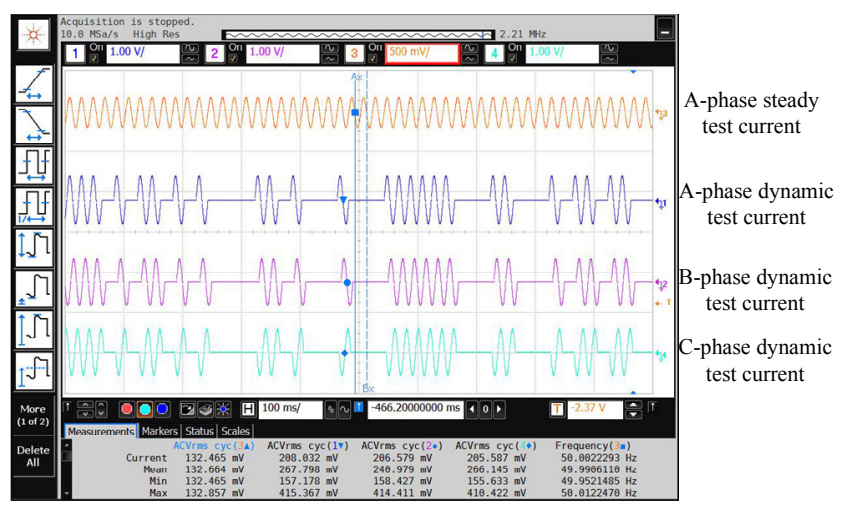

Fig.5. Waveforms of three-phase dynamic test current.

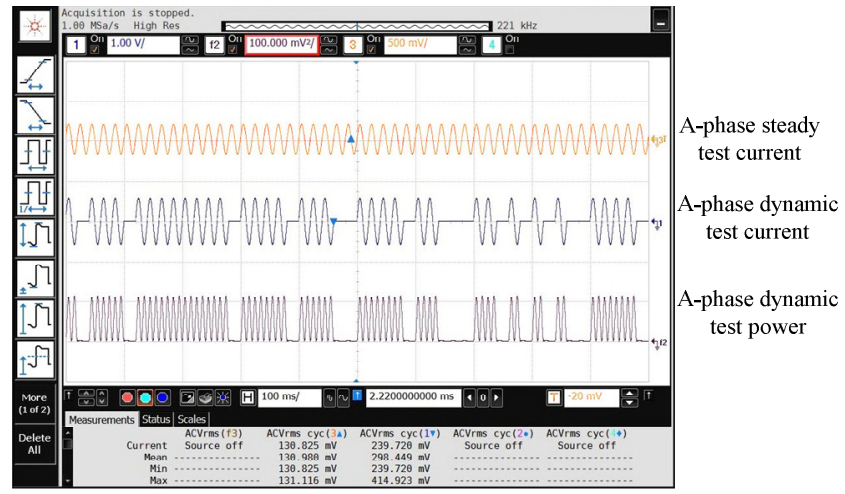

Fig.6. Waveforms of A-phase dynamic test current and power.

Fig.4. shows the simplicity of the keying implementation scheme. Fig.5. and Fig.6. illustrate that the m-sequence dynamic test signals can be generated effectively.

\section{SPARSENESS ANALYSIS OF PSEUDORANDOM DYNAMIC TEST POWER}

Based on the CM theory, the processed signal must satisfy the prerequisite of sparseness. In this section, the sparseness of $\mathrm{m}$-sequence dynamic test power is proved as follows.

As shown in (6), the steady test power $p_{k}^{s}(t)$ can be rewritten as

$$
p_{k}^{s}(t)=i_{k}^{s}(t) \cdot u_{k}^{s}(t)=\frac{I_{k} U_{k}}{2}\left[\cos \left(\Delta_{k}\right)-\cos \left(2 \Omega_{1} t+\theta_{k}\right)\right]
$$

where $\Delta_{k}=\varphi_{k}-\phi_{k}$ and $\theta_{k}=\varphi_{k}+\phi_{k}$. 
The autocorrelation function of $p_{k}^{s}(t)$ is calculated as (see (A1) in Appendix)

$$
R_{k}^{s}(\tau)=\frac{1}{T_{m}} \int_{0}^{T_{m}} p_{k}^{s}(t) p_{k}^{s}(t+\tau) d t=\frac{I_{k}^{2} U_{k}^{2}}{4} \cos ^{2}\left(\Delta_{k}\right)+\frac{I_{k}^{2} U_{k}^{2}}{8} \cos \left(2 \Omega_{1} \tau\right)
$$

Therefore, the autocorrelation function of $p_{k}^{d}(t)$ is (see (B1) in Appendix)

$$
\begin{aligned}
R_{k}^{d}(\tau) & =\frac{1}{T_{m}} \int_{0}^{T_{m}} p_{k}^{d}(t) p_{k}^{d}(t+\tau) d t \\
& =\frac{1}{T_{m}} \int_{0}^{T_{m}}\left[m(t) m(t+\tau) p_{k}^{s}(t) p_{k}^{s}(t+\tau)\right] d t \\
& =\left[\frac{I_{k}^{2} U_{k}^{2}}{4} \cos ^{2}\left(\Delta_{k}\right)+\frac{I_{k}^{2} U_{k}^{2}}{8} \cos \left(2 \Omega_{1} \tau\right)\right] \cdot \frac{1}{T_{m}} \int_{0}^{T_{m}} m(t) m(t+\tau) d t \\
& =R_{k}^{s}(\tau) R_{m}(\tau)
\end{aligned}
$$

where $R_{m}(\tau)$ is the autocorrelation function of $m(t)$.

According to the Parseval's theorem, the power spectral density is the Fourier transform of autocorrelation function, consequently, the power spectral density of $p_{k}^{d}(t)$ is

$$
\begin{aligned}
S_{k}^{d}(\Omega) & =\int_{-\infty}^{\infty} R_{k}^{d}(\tau) e^{-j \Omega \tau} d \tau=\int_{-\infty}^{\infty} R_{k}^{s}(\tau) R_{m}(\tau) e^{-j \Omega \tau} d \tau \\
& =\int_{-\infty}^{\infty}\left[\frac{I_{k}^{2} U_{k}^{2}}{4} \cos ^{2}\left(\Delta_{k}\right)+\frac{I_{k}^{2} U_{k}^{2}}{8} \cos \left(2 \Omega_{1} \tau\right)\right] R_{m}(\tau) e^{-j \Omega \tau} d \tau \\
& =\frac{I_{k}^{2} U_{k}^{2}}{4} \cos ^{2}\left(\Delta_{k}\right) S_{m}(\Omega)+\frac{I_{k}^{2} U_{k}^{2}}{8} \cdot \frac{1}{2 \pi}\left[S_{m}(\Omega) \otimes \int_{-\infty}^{\infty} \cos \left(2 \Omega_{1} \tau\right) e^{-j \Omega \tau} d \tau\right] \\
& =\frac{I_{k}^{2} U_{k}^{2}}{4} \cos ^{2}\left(\Delta_{k}\right) S_{m}(\Omega)+\frac{I_{k}^{2} U_{k}^{2}}{16}\left[S_{m}\left(\Omega-2 \Omega_{1}\right)+S_{m}\left(\Omega+2 \Omega_{1}\right)\right]
\end{aligned}
$$

where $S_{m}(\Omega)$ is the power spectral density of $m(t)$, which is expressed as [40]

$$
\begin{aligned}
S_{m}(\Omega) & =\int_{-\infty}^{\infty} R_{m}(\tau) e^{-j \Omega \tau} d \tau \\
& =\left(\frac{\pi}{2}+\frac{\pi}{N}+\frac{1}{4 N^{2}}\right) \delta(\Omega)+\frac{N+1}{4 N^{2}}\left[\frac{\sin (\Omega T / 2)}{(\Omega T / 2)}\right]_{\substack{l=-\infty \\
l \neq 0}}^{2} \delta\left(\Omega-\frac{\Omega_{1} l}{N}\right)
\end{aligned}
$$

Substituting (11) into (10), the power spectral density of $p_{k}^{d}(t)$ is rewritten as

$$
\begin{aligned}
& S_{k}^{d}(\Omega)= \frac{I_{k}^{2} U_{k}^{2}}{4} \cos ^{2}\left(\Delta_{k}\right) S_{m}(\Omega)+\frac{I_{k}^{2} U_{k}^{2}}{16}\left[S_{m}\left(\Omega-2 \Omega_{1}\right)+S_{m}\left(\Omega+2 \Omega_{1}\right)\right] \\
&=\left(\frac{\pi}{2}+\frac{\pi}{N}+\frac{1}{4 N^{2}}\right) \frac{I_{k}^{2} U_{k}^{2}}{16}\left[4 \pi \cos ^{2}\left(\Delta_{k}\right) \delta(\Omega)+\delta\left(\Omega-2 \Omega_{1}\right)+\delta\left(\Omega+2 \Omega_{1}\right)\right] \\
&+\frac{I_{k}^{2} U_{k}^{2}}{16 N^{2}}(N+1) \cos ^{2}\left(\Delta_{k}\right)\left[\frac{\sin \frac{\Omega T}{2}}{\frac{\Omega T}{2}}\right]_{\substack{l=-\infty \\
l \neq 0}}^{\infty} \delta\left(\Omega-\frac{\Omega_{1} l}{N}\right)+\frac{I_{k}^{2} U_{k}^{2}}{16} \frac{N+1}{4 N^{2}} \times \\
& \sum_{\substack{l=-\infty \\
l \neq 0}}^{\infty}\left\{\left[\frac{\sin \frac{\left(\Omega-2 \Omega_{1}\right) T}{2}}{2}\right]^{2} \delta\left(\Omega-2 \Omega_{1}-\frac{\Omega_{1} l}{N}\right)+\left[\frac{\sin \frac{\left(\Omega+2 \Omega_{1}\right) T}{2}}{\frac{\left(\Omega+2 \Omega_{1}\right) T}{2}}\right]^{2} \delta\left(\Omega+2 \Omega_{1}-\frac{\Omega_{1} l}{N}\right)\right\}
\end{aligned}
$$

By simulation using MATLAB, the autocorrelation functions and power spectral density functions of steady test power $p_{k}^{s}(t)$, m-sequence $m(t)$, and dynamic test power $p_{k}^{d}(t)$ are shown in Fig.7. The parameters for simulation are listed in Table 1.
Table 1. Parameters for simulation in Fig.7.

\begin{tabular}{|c|c|c|c|}
\hline Parameters & Value & Parameters & Value \\
\hline$k$ & $a$ & $f_{1}$ & $50 \mathrm{~Hz}$ \\
\hline$U_{a}$ & $220 \mathrm{~V}$ & $f_{s}$ & $\begin{array}{c}6000 \\
\mathrm{~Hz}\end{array}$ \\
\hline$I_{a}$ & $5 \mathrm{~A}$ & $\phi_{a}$ & $0^{\circ}$ \\
\hline$\varphi_{a}$ & $0^{\circ}$ & $T$ & $0.02 \mathrm{~s}$ \\
\hline $\begin{array}{c}\text { Length of } \\
\text { m-sequence } N\end{array}$ & 1023 & Duration $N T$ & $20.46 \mathrm{~s}$ \\
\hline
\end{tabular}
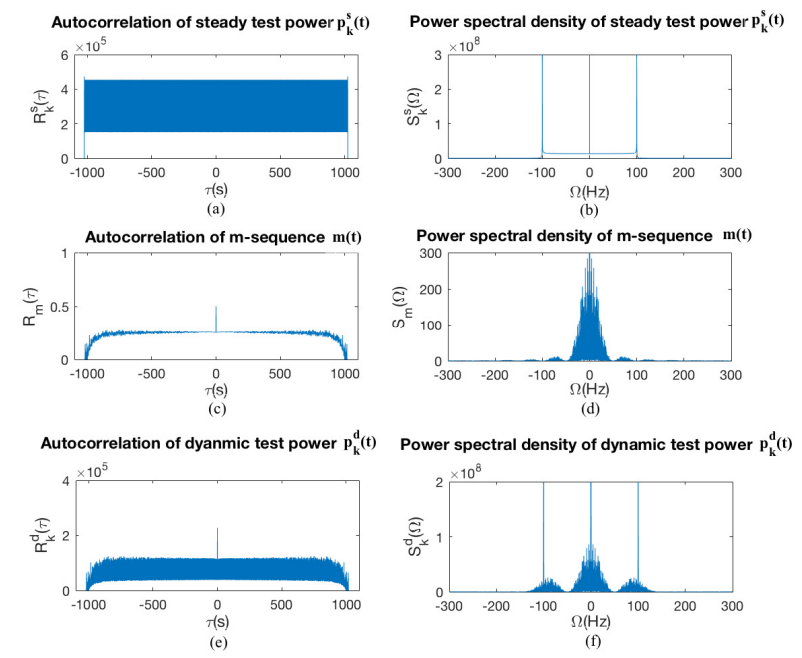

Fig.7. Autocorrelation functions and power spectral density functions of steady test power, m-sequence and dynamic test power.

Fig.7.a) and Fig.7.b) present that the steady test power is auto correlative, but the power spectrum is sparseness. Fig.7.c) and Fig.e) indicate that both autocorrelation function $R_{m}(\tau)$ and $R_{k}^{d}(\tau)$ are similar to the Dirichlet function, which show the approximate Gaussian distributions of m-sequence and dynamic test power besides amplitude random fluctuation and quasi-periodic change characteristics, this proves that the characteristics of dynamic test power are consistent with that of dynamic loads, as mentioned in Section 2.

For the power spectral density, as shown in (12) and Fig.7.f), the power spectrum of the dynamic test power $p_{k}^{d}(t)$ is discrete spectrum and sparseness in frequency domain. The sparse base is FFT base.

Therefore, it is concluded that the $\mathrm{CM}$ algorithm is suitable for measuring the electrical energy of m-sequence dynamic test power.

\section{ELECTRICAL ENERGY COMPRESSIVE MEASUREMENT ALGORITHM}

\section{A. Electrical energy CM algorithm model}

The discrete expression of $p_{k}^{d}(t)$ in (6) can be written as

$$
\begin{aligned}
p_{k}^{d}(n) & =\frac{I_{k} U_{k}}{2} m\left(n T_{s}\right)\left[\cos \left(\Delta_{k}\right)-\cos \left(2 \Omega_{1} n T_{s}+\theta_{k}\right)\right] \\
& =\frac{I_{k} U_{k}}{2} m\left(n T_{s}\right)\left[\cos \left(\Delta_{k}\right)-\cos \left(2 \omega_{1} n+\theta_{k}\right)\right]
\end{aligned}
$$


where $\omega_{1}=\Omega_{1} T_{s}$ is the digital frequency, $T_{s}=1 / f_{s}, f_{s}$ is the sampling frequency, $N_{s}$ is the number of sampling points in a fundamental period $T$, and $N_{s}=f_{s} / f_{1}, n$ is an integer number and $n=1,2, \cdots, N \cdot N_{s}$.

Let $N_{m}$ be the total number of sampling points in a msequence cyclical period $T_{m}$ and $N_{m}=N \cdot N_{s}$. The dynamic test power vector is defined as $\mathbf{P}_{k}^{d}=\left[p_{k}^{d}(1), p_{k}^{d}(2), \ldots p_{k}^{d}(n), \ldots, p_{k}^{d}\left(N_{m}\right)\right]$, which is regarded as the original processed signal of a $\mathrm{CM}$ matrix, the electrical energy of $\mathbf{P}_{k}^{d}$ is calculated by constructing an optimal sensing matrix $\boldsymbol{\Phi}_{o p}$.

Based on the CM theory, a general CM algorithm for calculating electrical energy of $\mathbf{P}_{k}^{d}$ can be expressed as

$$
\mathbf{E}_{k}^{q}=\boldsymbol{\Phi} \cdot \mathbf{P}_{k}^{d}
$$

where $\mathbf{E}_{k}^{q}$ is the electrical energy measurement vector, $q$ is an integer and $\mathbf{E}_{k}^{q}=\left[\begin{array}{llll}E_{k}(1) & E_{k}(2) & \cdots & E_{k}(q)\end{array}\right]$.

\section{B. Construction of optimal sensing matrix}

In order to accurately measure the electrical energy, a novel sensing matrix $\boldsymbol{\Phi}_{o p}$ for the CM algorithm is constructed through the system transfer function.

Let $\mathbf{h}_{1 \times N_{m}}=\left[h(1), h(2), \cdots, h\left(N_{m}\right)\right]$ be a system impulse response vector and $\mathbf{P}_{k}^{d}$ be a signal input to the system $\mathbf{h}_{1 \times N_{m}}$, the system output can be expressed as the convolution

$$
E_{k}(n)=h(n) \otimes p_{k}^{d}(n): \mathrm{n}=1,2, \cdots
$$

where $E_{k}(n)$ is the discrete electrical energy in the time interval $\left[0, n T_{s}\right]$.

According to the definition of convolution operation, equation (15) can be described as two matrices

\begin{tabular}{|c|c|c|c|c|c|c|c|c|c|}
\hline$E_{k}(1)$ & & $h(1)$ & 0 & 0 & $\cdots$ & 0 & 0 & 0 & \\
\hline$E_{k}(2)$ & & $h(2)$ & $h(1)$ & 0 & $\ldots$ & 0 & 0 & 0 & \\
\hline$E_{k}(3)$ & & $h(3)$ & $h(2)$ & $h(1)$ & $\ldots$ & 0 & 0 & 0 & $p_{k}^{d}(1)$ \\
\hline$\vdots$ & & $\vdots$ & $\vdots$ & $\vdots$ & $\ddots$ & $\vdots$ & $\vdots$ & $\vdots$ & $p_{k}^{d}(2)$ \\
\hline$E_{k}\left(N_{m}\right)$ & $=$ & $h\left(N_{m}\right)$ & $h\left(N_{m}-1\right)$ & $h\left(N_{m}-2\right)$ & $\cdots$ & $h(3)$ & $h(2)$ & $h(1)$ & $p_{k}^{d}(3)$ \\
\hline$E_{k}\left(N_{m}+1\right)$ & & 0 & $h\left(N_{m}\right)$ & $h\left(N_{m}-1\right)$ & $\cdots$ & $h(4)$ & $h(3)$ & $h(2)$ & $\vdots$ \\
\hline$E_{k}\left(N_{m}+2\right)$ & & 0 & 0 & $h\left(N_{m}\right)$ & $\cdots$ & $h(5)$ & $h(4)$ & $h(3)$ & $p_{k}^{d}\left(N_{m}\right)$ \\
\hline$\vdots$ & & $\vdots$ & $\vdots$ & $\vdots$ & $\ddots$ & $\vdots$ & $\vdots$ & $\vdots$ & \\
\hline$E_{k}\left(2 N_{m}-1\right)$ & & 0 & 0 & 0 & $\cdots$ & 0 & 0 & $h\left(N_{m}\right)$ & \\
\hline$\Downarrow$ & & & & $\Downarrow$ & & & & & $\Downarrow$ \\
\hline $\mathbf{E}_{k}^{2 N_{m}-1}$ & & & & $\Phi$ & & & & & \\
\hline
\end{tabular}
multiplication

Meanwhile, equation (16) can be simplified as

$$
\mathbf{E}_{k}^{2 N_{m}-1}=\boldsymbol{\Phi} \cdot \mathbf{P}_{k}^{d}
$$

Equation (17) presents that the general sensing matrix $\boldsymbol{\Phi}$ is composed of elements $h(n)$. Because the aim of the CM algorithm is to retain the maximum average electrical energy of signal $p_{k}^{d}(n)$, from (16), the maximum energy $E_{k}^{c m}$ calculated by the CM matrix can be obtained from the maximum element of vector $\mathbf{E}_{k}^{2 N_{m}-1}$ as

$$
\begin{aligned}
E_{k}^{c m} & =\max _{n}\left\{E_{k}(n)\right\}=E_{k}^{N_{m}} \\
& =\left[\begin{array}{llll}
h\left(N_{m}\right) & h\left(N_{m}-1\right) & \ldots & h(1)
\end{array}\right]\left[\begin{array}{c}
p_{k}^{d}(1) \\
p_{k}^{d}(2) \\
\vdots \\
p_{k}^{d}\left(N_{m}\right)
\end{array}\right]
\end{aligned}
$$

Equation (18) can be rewritten in a simple form as

$$
E_{k}^{c m}=\boldsymbol{\Phi}_{o p} \cdot \mathbf{P}_{k}^{d}
$$

Equation (19) means that the constructed novel sensing matrix $\mathbf{\Phi}_{o p}=\left[\phi_{o p}(n)\right]_{1 \times N_{m}}=\left[h\left(N_{m}-n+1\right)\right]_{1 \times N_{m}}$, which is a vector form with $1 \times N_{m}$ and minimum measurement error. From (15) and (17), the optimal sensing matrix elements of $\boldsymbol{\Phi}_{o p}$ can be designed by $h(n)$ through system steady-state optimization method.

By the discrete-time Fourier transform (DTFT) of (15), the frequency domain form of electrical energy is:

$$
E_{k}^{c m}(\omega)=H(\omega) P_{k}^{d}(\omega)
$$

where $H(\omega)$ is the DTFT of $h(n), P_{k}^{d}(\omega)$ is the DTFT of $p_{k}^{d}(n)$, which is calculated as

$$
\begin{gathered}
P_{k}^{d}(\omega)=\sum_{n=1}^{N_{m}} p_{k}^{d}(n) e^{-j \omega n}=\pi I_{k} U_{k} \cos \left(\Delta_{k}\right) \sum_{i=-\infty}^{+\infty} \delta(\omega-2 \pi i) \\
-\frac{I_{k} U_{k}}{2} \pi\left[e^{-j \theta_{k}} \sum_{i=-\infty}^{+\infty} \delta\left(\omega+2 \omega_{1}-2 \pi i\right)+e^{j j_{k}} \sum_{i=-\infty}^{+\infty} \delta\left(\omega-2 \omega_{1}-2 \pi i\right)\right] \\
H(\omega)=\sum_{n=1}^{N_{m}} h(n) e^{-j \omega n}
\end{gathered}
$$

According to the steady-state optimization method, the $H(\omega)$ is defined as an optimization variable, the objective function and constraint condition in the frequency domain are as follows

$$
J(\omega)=C_{1} \sum_{n=1}^{N_{m}}\left[E_{k}^{c m}(\omega)-E_{k}^{s}(\omega)\right]+C_{2} \sum_{n=1}^{N_{m}} H^{2}(\omega)
$$

$$
\text { s.t. } E_{k}^{s}(\omega)=\sum_{n=1}^{N_{m}} E_{k}^{s} e^{-j \omega n}=\sum_{n=1}^{N_{m}} \frac{I_{k} U_{k} T_{m}}{2} \cos \left(\Delta_{k}\right) e^{-j \omega n}
$$

where $E_{k}^{s}$ is the theoretical steady electrical energy and $E_{k}^{s}=\frac{I_{k} U_{k} T_{m}}{2} \cos \left(\Delta_{k}\right)$, the minimum value of performance index $J$ is calculated as

$$
\frac{\partial J}{\partial H}=C_{1} P_{k}^{d}(\omega)+2 C_{2} H(\omega)=0
$$


where $C_{1}$ and $C_{2}$ are constants, therefore, the general form of $H(\omega)$ in frequency domain is

$$
\begin{aligned}
H(\omega)= & -\frac{C_{1}}{2 C_{2}} P_{k}^{d}(\omega)=-\frac{C_{1}}{2 C_{2}} \pi I_{k} U_{k} \cos \left(\Delta_{k}\right) \sum_{i=-\infty}^{+\infty} \delta(\omega-2 \pi i) \\
& +\frac{C_{1} I_{k} U_{k} \pi}{4 C_{2}}\left[e^{-j \theta_{k}} \sum_{i=-\infty}^{+\infty} \delta\left(\omega+2 \omega_{1}-2 \pi i\right)+e^{j \theta_{k}} \sum_{i=-\infty}^{+\infty} \delta\left(\omega-2 \omega_{1}-2 \pi i\right)\right]
\end{aligned}
$$

It is defined $C=-C_{1} I_{k} U_{k} / 4 C_{2}$ as a constant, when $\Delta_{k}=\theta_{k}=0$, equation (25) can be rewritten as:

$$
\begin{aligned}
H(\omega)=C & {\left[2 \pi \sum_{i=-\infty}^{+\infty} \delta(\omega-2 \pi i)-\pi \sum_{i=-\infty}^{+\infty} \delta\left(\omega+2 \omega_{1}-2 \pi i\right)\right.} \\
- & \left.\pi \sum_{i=-\infty}^{+\infty} \delta\left(\omega-2 \omega_{1}-2 \pi i\right)\right]
\end{aligned}
$$

By the inverse DTFT of $H(\omega)$, the general form of $h(n)$ is

$$
h(n)=\operatorname{IDTFT}[H(\omega)]=C\left[1-\cos \left(2 \omega_{1} n\right)\right]
$$

As mentioned before, $\omega_{1}=\Omega_{1} T_{s}=2 \pi f_{1} / f_{s}$, the $h(n)$ can be also expressed as

$$
h(n)=\operatorname{IDTFT}[H(\omega)]=C\left[1-\cos \left(\frac{4 \pi f_{1} n}{f_{s}}\right)\right]
$$

Meanwhile, $\phi_{o p}(n)=h\left(N_{m}-n+1\right)$, the general form of $\phi_{o p}(n)$ is

$$
\begin{aligned}
\phi_{o p}(n) & =h\left(N_{m}-n+1\right)=C\left[1-\cos \left(\frac{4 \pi f_{1}\left(N_{m}-n+1\right)}{f_{s}}\right)\right] \\
& =C\left\{1-\cos \left[\frac{4 \pi f_{1}(n-1)}{f_{s}}\right]\right\}
\end{aligned}
$$

Therefore, $\boldsymbol{\Phi}_{o p}=\left[\phi_{o p}(n)\right]_{1 \times N_{m}}$ is the optimal sensing matrix with minimum error.

Finally, the mathematical expression for the calculation of electrical energy using the $\mathrm{CM}$ algorithm is:

$$
\begin{aligned}
E_{k}^{c m} & =\boldsymbol{\Phi}_{o p} \cdot \mathbf{P}_{k}^{d}=\left[\phi_{o p}(n)\right]_{1 \times N_{m}} \cdot\left\{\left[p_{k}^{d}(n)\right]^{\mathrm{T}}\right\}_{N_{m} \times 1} \\
& =\left[\phi_{o p}(1) \phi_{o p}(2) \ldots \phi_{o p}\left(N_{m}\right)\right] \cdot\left[\begin{array}{c}
p_{k}^{d}(1) \\
p_{k}^{d}(2) \\
\vdots \\
p_{k}^{d}\left(N_{m}\right)
\end{array}\right]=\sum_{n=1}^{N_{m}} \phi_{o p}(n) p_{k}^{d}(n)
\end{aligned}
$$

The accuracy of the CM algorithm is evaluated by the relative error of $E_{k}^{c m}$ given in

$$
e=\frac{\left|E_{k}^{c m}-E_{k}^{d}\right|}{E_{k}^{d}}
$$

where $E_{k}^{d}$ is the reference theoretical electrical energy, which is calculated as

$$
E_{k}^{d}=N_{o n} \int_{0}^{T} p_{k}^{d}(t) d t
$$

where $N_{o n}$ is the number of $m(r)=1$ in a cyclical period of m-sequence.

\section{RIP proof of optimal sensing matrix $\boldsymbol{\Phi}_{o p}$}

As mentioned before, the sensing matrix used for the CM algorithm must meet the restricted isometry property (RIP). In [18], Candes proved that the RIP is equivalent to the fact that the sensing matrix is uncorrelated to the sparse base. In this section, the $\boldsymbol{\Phi}_{o p}$ is proved to satisfy the RIP.

In Section 3, the FFT base is used as sparse base:

$$
\Psi=\frac{1}{\sqrt{N_{m}}} \mathbf{W}_{N_{m}}
$$

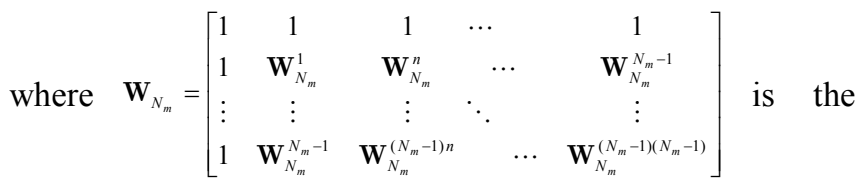
transformation matrix, $\mathbf{W}_{N_{m}}=e^{-j \frac{2 \pi}{N_{m}}}$.

The correlation of $\boldsymbol{\Phi}_{o p}$ and $\boldsymbol{\Psi}$ is calculated according to the minimum rank of $\left[\begin{array}{c}\boldsymbol{\Phi}_{o p} \\ \boldsymbol{\Psi}\end{array}\right]_{\left(N_{m}+1\right) \times N_{m}}$ :

$$
\left[\begin{array}{c}
\boldsymbol{\Phi}_{o p} \\
\Psi
\end{array}\right]_{\left(N_{m}+1\right) \times N_{m}}=\left[\begin{array}{cccc}
1 & 1-\cos 2 \omega_{1} & \cdots & 1-\cos 2 \omega_{1}\left(N_{m}-1\right) \\
1 & 1 & \cdots & 1 \\
1 & \mathbf{W}_{N_{m}}^{1} & \cdots & \mathbf{W}_{N_{m}}^{N_{m}-1} \\
\vdots & \vdots & \ddots & \vdots \\
1 & \mathbf{W}_{N_{m}}^{N_{m}-1} & \cdots & \mathbf{W}_{N_{m}}^{\left(N_{m}-1\right)\left(N_{m}-1\right)}
\end{array}\right]
$$

after element transformation to get:

$$
\left[\begin{array}{c}
\boldsymbol{\Phi}_{o p} \\
\Psi
\end{array}\right]_{\left(N_{m}+1\right) \times N_{m}}=\left[\begin{array}{cccc}
1 & 1 & \cdots & 1 \\
0 & \cos 2 \omega_{1} & \cdots & \cos 2 \omega_{1}\left(N_{m}-1\right) \\
0 & 1-\mathbf{W}_{N_{m}}^{1} & \cdots & 1-\mathbf{W}_{N_{m}-1}^{N_{m}} \\
\vdots & \vdots & \ddots & \vdots \\
0 & 1-\mathbf{W}_{N_{m}}^{N_{m}-1} & \cdots & 1-\mathbf{W}_{N_{m}}^{\left(N_{m}-1\right)\left(N_{m}-1\right)}
\end{array}\right]
$$

Obviously, the rand of $\left[\begin{array}{c}\boldsymbol{\Phi}_{o p} \\ \boldsymbol{\Psi}\end{array}\right]_{\left(N_{m}+1\right) \times N_{m}}$ is $N_{m}+1$, which proves that the $\left[\begin{array}{c}\Phi_{o p} \\ \Psi\end{array}\right]_{\left(N_{m}+1\right) \times N_{m}}$ is a row full rank matrix and each row in the matrix is uncorrelated, that is to say, the $\boldsymbol{\Phi}_{o p}$ is uncorrelated to the sparse base $\boldsymbol{\Psi}$. Therefore, the optimal sensing matrix with minimum error $\boldsymbol{\Phi}_{o p}$ satisfies the RIP property.

\section{SiMULATION TESTS AND RESULT DISCUSSION}

In order to evaluate the accuracy of the CM algorithm used for electrical energy measurement, using MATLAB, a number of simulation tests were carried out in respect to different influence factors. 


\section{A. Simulation of m-sequence dynamic test signals}

Firstly, simulation was carried out for the realization of msequence dynamic test signals. By setting parameters, the mathematical expressions of $\mathrm{m}$-sequence dynamic test signals are listed in the following:

$$
\begin{gathered}
u_{k}^{s}(t)=220 \sin (100 \pi t) \\
i_{k}^{d}(t)=m(t) i_{k}^{s}(t)=5 m(t) \sin (100 \pi t+\pi / 3) \\
p_{k}^{d}(t)=550 m(t)[1 / 2-\cos (100 \pi t+\pi / 3)]
\end{gathered}
$$

Other simulation conditions are as follows.

1) The sampling frequency is $6000 \mathrm{~Hz}$.

2) The length of $m$-sequence in a cyclical period is 31 .

3) The number of cyclical periods $M$ is 2 and simulation duration is $1.24 \mathrm{~s}$.
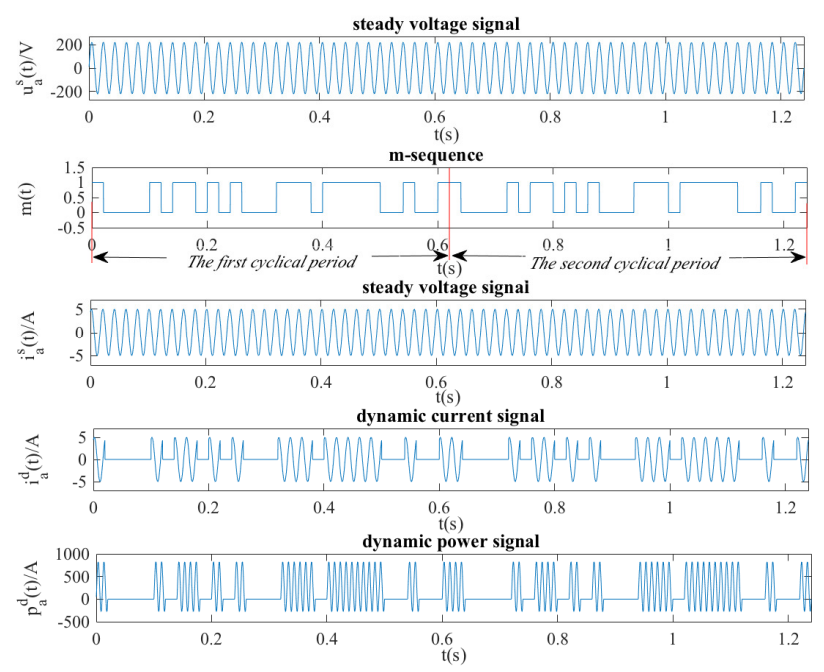

Fig.8. Simulation waveforms of dynamic test signals.
The obtained waveforms are shown in Fig.8., which contains steady test voltage and current, m-sequence, dynamic test current and power. It can be observed that the dynamic test current and power have the same characteristics as $\mathrm{m}$-sequence. As mentioned before, the characteristics are consistent with actual dynamic loads.

\section{B. Accuracy of CM algorithm under different dynamic test signal conditions}

For different $\mathrm{m}$-sequence dynamic test signals, the errors of the CM algorithm were evaluated by simulations. The common simulation test conditions are as follows:

1) The fundamental frequency $f_{1}=50 \mathrm{~Hz}$.

2) $U_{k}=220 \mathrm{~V}$ and $I_{k}=5 \mathrm{~A}$.

1) Different lengths $N$ of m-sequence in a cyclical period

Firstly, simulations were performed with different lengths of $\mathrm{m}$-sequence in a cyclical period. The cyclical period number was set to 3 and sampling frequency was $6000 \mathrm{~Hz}$. The errors of the CM algorithm are reported in Table 2.

2) Different cyclical period numbers $M$ of m-sequence

Secondly, simulations were performed with different cyclical period numbers of $\mathrm{m}$-sequence. The length of $\mathrm{m}$ sequence in a cyclical period was set to 1023 and sampling frequency was $6000 \mathrm{~Hz}$. The errors of the $\mathrm{CM}$ algorithm are reported in Table 3.

3) Different sampling frequency

In addition, simulations were performed with different sampling frequency. The $N=1023$ and $M=3$, the power factor was 1.0 and simulation signal time duration was $61.38 \mathrm{~s}$. The errors of the $\mathrm{CM}$ algorithm are reported in Table 4.

As for the results from Table 2.-Table 4., it can be proved that with different test conditions, the errors of the $\mathrm{CM}$ algorithm are superior to $1 \times 10^{-13}$. Therefore, the $\mathrm{CM}$ algorithm shows very good performance for the electrical energy measurement of $\mathrm{m}$-sequence dynamic test signals.

Table 2. Errors of CM algorithm under different lengths of m-sequence dynamic test signals.

\begin{tabular}{|c|c|c|c|c|c|}
\hline $\begin{array}{l}\text { Power } \\
\text { factor }\end{array}$ & $\begin{array}{l}\text { Length of } \\
\text { m-sequence }\end{array}$ & $\begin{array}{l}\text { Duration } \\
{[\mathbf{s}]}\end{array}$ & $\begin{array}{l}\text { Theoretical electrical } \\
\text { energy [Ws] }\end{array}$ & $\begin{array}{l}\text { Electrical energy calculated } \\
\text { by CM algorithm [Ws] }\end{array}$ & $\begin{array}{l}\text { Error } \\
{\left[\times 10^{-14}\right]}\end{array}$ \\
\hline \multirow{2}{*}{$\begin{array}{c}1.0 \\
\varphi_{k}=0^{\circ}\end{array}$} & 127 & 7.62 & 2112.000000000001 & 2111.9999999999920 & 3.81 \\
\cline { 2 - 6 }$\phi_{k}=0^{\circ}$ & 255 & 15.3 & 4224.000000000001 & 4223.9999999999830 & 4.00 \\
\cline { 2 - 6 } & 511 & 30.66 & 8448.000000000002 & 8448.000000000724 & 7.47 \\
\hline \multirow{2}{*}{$0.5 \mathrm{~L}$} & 1023 & 61.38 & 16896.00000000000 & 16895.999999999779 & 11.7 \\
\cline { 2 - 6 }$\varphi_{k}=0^{\circ}$ & 127 & 7.62 & 1056.000000000000 & 1056.0000000000040 & 3.81 \\
\cline { 2 - 6 }$\phi_{k}=60^{\circ}$ & 511 & 15.3 & 2112.000000000000 & 2112.0000000000084 & 3.95 \\
\cline { 2 - 6 } & 1023 & 61.38 & 4223.999999999999 & 4224.000000000464 & 9.32 \\
\hline $0.8 \mathrm{C}$ & 127 & 7.62 & 1689.5997736249649 & 8447.9999999999020 & 11.0 \\
\cline { 2 - 6 }$\varphi_{k}=36.87^{\circ}$ & 255 & 15.3 & 3379.195472499298 & 3379.195476249695 & 2.69 \\
\cline { 2 - 6 }$\phi_{k}=0^{\circ}$ & 511 & 30.66 & 6758.390944998597 & 6758.390944999350 & 1.77 \\
\cline { 2 - 6 } & 1023 & 61.38 & 13516.78188999719 & 13516.781889999792 & 1.21 \\
\hline
\end{tabular}


Table 3. Errors of CM algorithm under different cyclical period numbers of m-sequence dynamic test signals.

\begin{tabular}{|c|l|l|l|c|c|}
\hline $\begin{array}{c}\text { Power } \\
\text { factor }\end{array}$ & $\begin{array}{l}\text { Cyclical period numbers } \\
\text { of m-sequence }\end{array}$ & $\begin{array}{l}\text { Duration } \\
{[\mathbf{s}]}\end{array}$ & $\begin{array}{l}\text { Theoretical } \\
\text { electrical energy } \\
{[\mathbf{W}]}\end{array}$ & $\begin{array}{l}\text { Electrical energy } \\
\text { calculated by } \\
\text { CM algorithm [Ws] }\end{array}$ & $\begin{array}{l}\text { Error } \\
{\left[\times 10^{-14}\right]}\end{array}$ \\
\hline \multirow{2}{*}{$\begin{array}{c}1.0 \\
\varphi_{k}=0^{\circ}\end{array}$} & 1 & 20.46 & 5632.000000000001 & 5631.999999999664 & 5.98 \\
\cline { 2 - 7 }$\phi_{k}=0^{\circ}$ & 2 & 40.92 & 11264.00000000000 & 11264.00000000170 & 13.3 \\
\cline { 2 - 7 } & 3 & 61.38 & 16896.00000000000 & 16895.99999999779 & 13.2 \\
\hline \multirow{2}{*}{$0.5 \mathrm{~L}$} & 4 & 81.84 & 22528.00000000000 & 22527.99999999419 & 19.9 \\
\cline { 2 - 7 }$\varphi_{k}=0^{\circ}$ & 1 & 20.46 & 2816.000000000000 & 2816.000000000085 & 3.02 \\
\cline { 2 - 7 }$\phi_{k}=60^{\circ}$ & 2 & 40.92 & 5631.999999999999 & 5632.000000000822 & 12.3 \\
\hline \multirow{2}{*}{$0.8 \mathrm{C}$} & 3 & 61.38 & 8447.999999999998 & 8447.999999999020 & 11.8 \\
\cline { 2 - 7 }$\varphi_{k}=36.87^{\circ}$ & 4 & 81.84 & 11264.00000000000 & 11263.99999999747 & 17.5 \\
\cline { 2 - 7 }$\phi_{k}=0^{\circ}$ & 1 & 40.46 & 4505.593963332399 & 4505.593963332376 & 0.505 \\
\cline { 2 - 7 } & 2 & 61.38 & 13516.78188999719 & 13516.78188999792 & 4.48 \\
\hline
\end{tabular}

Table 4. Errors of $\mathrm{CM}$ algorithm under different cyclical sampling frequency.

\begin{tabular}{|c|c|c|c|}
\hline $\begin{array}{l}\text { Sampling frequency } \\
{[\mathbf{H z}]}\end{array}$ & $\begin{array}{l}\text { Theoretical electrical energy } \\
{[\mathbf{W}]}\end{array}$ & $\begin{array}{l}\text { Electrical energy calculated by } \\
\text { CM algorithm [Ws] }\end{array}$ & $\begin{array}{l}\text { Error } \\
{\left[\times 10^{-14}\right]}\end{array}$ \\
\hline 6000 & 16896.00000000000 & 16895.99999999779 & 13.1 \\
\hline 5500 & 16896.00000000000 & 16895.99999999933 & 8.54 \\
\hline 5000 & 16896.00000000000 & 16896.00000000369 & 13.0 \\
\hline 4500 & 16896.00000000001 & 16895.99999999816 & 12.5 \\
\hline 4000 & 16896.00000000000 & 16895.999999999993 & 10.1 \\
\hline 3500 & 16895.99999999999 & 16895.99999999925 & 9.10 \\
\hline 3000 & 16896.00000000000 & 16895.99999999968 & 8.08 \\
\hline 2500 & 16896.00000000000 & 16895.99999999998 & 7.09 \\
\hline 2000 & 16896.00000000000 & 16895.99999999773 & 7.80 \\
\hline 1500 & 16896.00000000000 & 16895.99999999974 & 7.17 \\
\hline 1000 & 16896.00000000000 & 16895.999999999769 & 7.76 \\
\hline
\end{tabular}

\section{Comparison against other window-based algorithms}

As we all know, the window-based algorithms are usually adopted to design low pass filters for filtering the input power and measuring electrical energy by accumulating the output power, which can be expressed as

$$
E_{k}^{w}=\sum_{n=1}^{N_{m}} \tilde{p}(n)
$$

where $\tilde{p}(n)$ is the output power of low pass filters.

Table 5. Coefficients $a_{d}$ of three cosine windows.

\begin{tabular}{|c|c|l|l|}
\hline Window & $a_{0}$ & $a_{1}$ & $a_{2}$ \\
\hline Rectangular & 1.00 & & \\
\hline Hanning & 0.50 & 0.50 & \\
\hline Blackman & 0.42 & 0.50 & 0.08 \\
\hline
\end{tabular}

The most popular windows are cosine windows, which are defined as

$$
w(v)=\sum_{d=0}^{D}(-1)^{2} a_{d} \cos \left(\frac{2 \pi d v}{N_{w}}\right), \quad v=0,1, \cdots, N_{w}-1
$$

where $N_{w}$ is the window length, $D$ is the number of window coefficients $a_{d}$ and $D \geq 1$. The coefficients $a_{d}$ of three kinds of proposed windows are listed in Table 5. [17].

The comparisons were performed between the CM algorithm and three window-based algorithms through two validation test cases and the common conditions are:

1) The sampling frequency is $1600 \mathrm{~Hz}$.

2) Cyclical periods $M=3$.

3) $U_{k}=220 \mathrm{~V}, I_{k}=5 \mathrm{~A}$ and power factor is 1.0.

4) The window length is 128 . 


\section{1) Different simulation durations}

Firstly, with different lengths of m-sequence in a cyclical period, comparisons were completed under several different simulation durations. The result is shown in Fig.9. As can be seen from the results, for the CM algorithm, the relative errors are always small and less than $1 \times 10^{-13}$, which are much smaller than those of other window-based algorithms.

2) Different fundamental frequency of dynamic test signals

Secondly, comparisons were completed with different fundamental frequency of dynamic test signals and the dynamic test conditions became nonsynchronized. The length of $\mathrm{m}$-sequence in a cyclical period is 1023 . The comparing result is shown in Table 6 .

The above results mean that the $\mathrm{CM}$ algorithm significantly outperforms the other window-based algorithms under nonsynchronous sampling conditions.

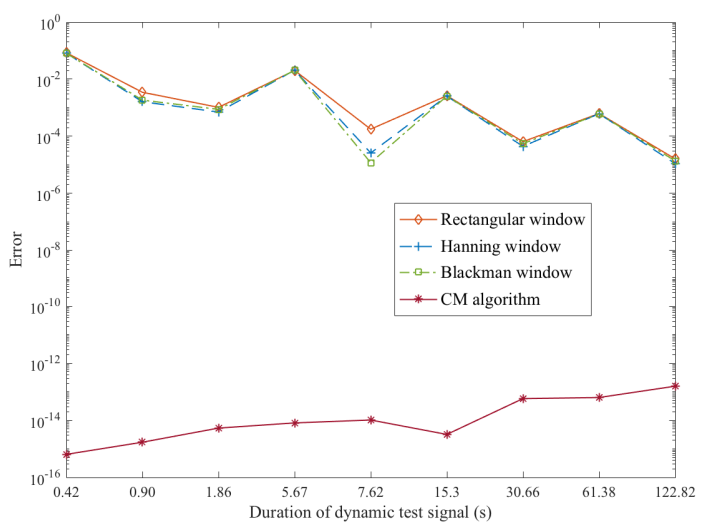

Fig.9. Errors of CM algorithm and other window-based algorithms.

Table 6. Errors of $\mathrm{CM}$ algorithm and other window-based algorithms.

\begin{tabular}{|c|c|c|c|c|c|}
\hline $\begin{array}{l}\text { Fundamental } \\
\text { frequency }[\mathbf{H z}]\end{array}$ & $\begin{array}{l}\text { CM algorithm } \\
{\left[\times 10^{-14}\right]}\end{array}$ & $\begin{array}{l}\text { Rectangular } \\
\text { window }\left[\times 10^{-4}\right]\end{array}$ & $\begin{array}{l}\text { Hanning } \\
\text { window }\left[\times 10^{-4}\right]\end{array}$ & $\begin{array}{l}\text { Blackman } \\
\text { window }\left[\times 10^{-4}\right]\end{array}$ & $\begin{array}{l}\text { Theoretical electrical } \\
\text { energy [Ws] }\end{array}$ \\
\hline 47.00 & 22.2 & 5.69 & 5.63 & 5.57 & 17951.99999999813 \\
\hline 47.50 & 12.7 & 5.97 & 5.89 & 5.82 & 17423.99999999776 \\
\hline 48.00 & 12.7 & 5.97 & 5.89 & 5.82 & 17423.99999999776 \\
\hline 48.50 & 6.37 & 6.22 & 6.12 & 6.05 & 16896.00000000000 \\
\hline 49.00 & 6.37 & 6.22 & 6.12 & 6.05 & 16896.00000000000 \\
\hline 49.50 & 6.37 & 6.22 & 6.12 & 6.05 & 16896.00000000000 \\
\hline 50.00 & 6.37 & 6.22 & 6.12 & 6.05 & 16896.00000000000 \\
\hline 50.50 & 16.7 & 6.44 & 6.31 & 6.23 & 16368.00000000254 \\
\hline 51.00 & 16.7 & 6.44 & 6.31 & 6.23 & 16368.00000000254 \\
\hline 51.50 & 16.7 & 6.44 & 6.31 & 6.23 & 16368.000000000254 \\
\hline 52.00 & 1.61 & 6.60 & 6.45 & 6.38 & 15839.999999999736 \\
\hline
\end{tabular}

\section{CONCLUSION}

In this paper, firstly, a novel three-phase m-sequence dynamic test current and power signals are proposed to reflect the main characteristics of actual dynamic loads in smart grid, a keying implementation scheme for the generation of the dynamic test signals is established, the effectiveness of the implementation scheme has been verified in experimental environment.

Secondly, using the steady-state optimization method, an optimal measurement matrix with minimum measurement error is designed. Moreover, a CM algorithm is proposed for accurate electrical energy measurement. Simulation results show that the maximum errors of the $\mathrm{CM}$ algorithm are always lower than $1 \times 10^{-13}$, which significantly outperforms that of another cosine window-based algorithm.

The works of this paper can be applied for testing the dynamic errors of electricity meters and modifying the algorithm in electricity meters to reduce their errors under dynamic current conditions.

\section{APPENDIX}

\section{A. Derivation of $R_{k}^{s}(\tau)$}

In (8), the specific calculation processes of the autocorrelation function $R_{k}^{s}(\tau)$ can be shown as (A1)

$$
\begin{aligned}
R_{k}^{s}(\tau) & =\frac{1}{T_{m}} \int_{0}^{T_{m}} p_{k}^{s}(t) p_{k}^{s}(t+\tau) d t=\frac{1}{T_{m}} \int_{0}^{T_{m}}\left[\frac{I_{k} U_{k}}{2} \cos \left(\Delta_{k}\right)-\frac{I_{k} U_{k}}{2} \cos \left(2 \Omega_{1} t+\theta_{k}\right)\right]\left[\frac{I_{k} U_{k}}{2} \cos \left(\Delta_{k}\right)-\frac{I_{k} U_{k}}{2} \cos \left(2 \Omega_{1} t+2 \Omega_{1} \tau+\theta_{k}\right)\right] d t \\
& =\frac{1}{T_{m}} \int_{0}^{T_{m}}\left[\begin{array}{l}
\left.\frac{I_{k}^{2} U_{k}^{2}}{4} \cos ^{2}\left(\Delta_{k}\right)-\frac{I_{k}^{2} U_{k}^{2}}{4} \cos \left(\Delta_{k}\right) \cos \left(2 \Omega_{1} t+2 \Omega_{1} \tau+\theta_{k}\right)-\frac{I_{k}^{2} U_{k}^{2}}{4} \cos \left(\Delta_{k}\right) \cos \left(2 \Omega_{1} t+\theta_{k}\right)\right] d t \\
+\frac{I_{k}^{2} U_{k}^{2}}{8} \cos \left(2 \Omega_{1} \tau\right)+\frac{I_{k}^{2} U_{k}^{2}}{8} \cos \left(4 \Omega_{1} t+2 \Omega_{1} \tau+2 \theta_{k}\right)
\end{array}\right] d t=\frac{I_{k}^{2} U_{k}^{2}}{4} \cos ^{2}\left(\Delta_{k}\right)+\frac{I_{k}^{2} U_{k}^{2}}{8} \cos \left(2 \Omega_{1} \tau\right) \\
& =\frac{1}{T_{m}} \int_{0}^{T_{m}}\left[\frac{I_{k}^{2} U_{k}^{2}}{4} \cos ^{2}\left(\Delta_{k}\right)+\frac{I_{k}^{2} U_{k}^{2}}{8} \cos \left(2 \Omega_{1} \tau\right)\right] d t=\frac{1}{8}
\end{aligned}
$$




\section{B. Derivation of $R_{k}^{d}(\tau)$}

In (9), the specific calculation processes of the autocorrelation function $R_{k}^{d}(\tau)$ can be shown as (B1)

$$
\begin{aligned}
& R_{k}^{d}(\tau)=\frac{1}{T_{m}} \int_{0}^{T_{m}} m(t) m(t+\tau) p_{k}^{s}(t) p_{k}^{s}(t+\tau) d t \\
& =\frac{1}{T_{m}} \int_{0}^{T_{m}} m(t) m(t+\tau)\left[\begin{array}{l}
\frac{I_{k}^{2} U_{k}^{2}}{4} \cos ^{2}\left(\Delta_{k}\right)-\frac{I_{k}^{2} U_{k}^{2}}{4} \cos \left(\Delta_{k}\right) \cos \left(2 \Omega_{1} t+2 \Omega_{1} \tau+\theta_{k}\right)-\frac{I_{k}^{2} U_{k}^{2}}{4} \cos \left(\Delta_{k}\right) \cos \left(2 \Omega_{1} t+\theta_{k}\right) \\
+\frac{I_{k}^{2} U_{k}^{2}}{8} \cos \left(2 \Omega_{1} \tau\right)+\frac{I_{k}^{2} U_{k}^{2}}{8} \cos \left(4 \Omega_{1} t+2 \Omega_{1} \tau+2 \theta_{k}\right)
\end{array}\right] d t \\
& =\frac{1}{T_{m}} \int_{0}^{T_{m}} m(t) m(t+\tau)\left[\frac{I_{k}^{2} U_{k}^{2}}{4} \cos ^{2}\left(\Delta_{k}\right)+\frac{I_{k}^{2} U_{k}^{2}}{8} \cos \left(2 \Omega_{1} \tau\right)\right] d t=\left[\frac{I_{k}^{2} U_{k}^{2}}{4} \cos ^{2}\left(\Delta_{k}\right)+\frac{I_{k}^{2} U_{k}^{2}}{8} \cos \left(2 \Omega_{1} \tau\right)\right] R_{m}(\tau)=R_{k}^{s}(\tau) R_{m}(\tau)
\end{aligned}
$$

\section{ACKNOWLEDGMENT}

This work was supported by the National Natural Science Foundation of China (No. NSFC-51577006).

\section{REFERENCES}

[1] Kukuča, P., Chrapčiak, I. (2016). From smart metering to smart grid. Measurement Science Review, 16 (3), 142-148.

[2] Lao, K.-W., Wong, M.-C., Dai, N., Wong, C.-K., Lam, C.-S. (2015). A systematic approach to hybrid railway power conditioner design with harmonic compensation for high-speed railway. IEEE Transactions on Industrial Electronics, 62 (2), 930-942.

[3] Bernieri, A., Betta, G., Ferrigno, L., Laracca, M., Moriello, R.S.L. (2013). Electrical energy metering: Some challenges of the European Directive on Measuring Instruments (MID). Measurement, 46 (9), 3347-3354.

[4] Artale, G., Cataliotti, A., Cosentino, V., Cara, D.D., Nuccio, S., Tinè, G. (2017). Arc fault detection method based on CZT low-frequency harmonic current analysis. IEEE Transactions on Instrumentation and Measurement, 66 (5), 2232 -2239.

[5] Wang, X.W., Chen, J.X., Yuan, R.M., Jia, X.L., Zhu, M., Jiang, Z.Y. (2017). OOK power model based dynamic error testing for smart electricity meter. Measurement Science and Technology, 28 (2), 025015.

[6] OIML. (2006). Instruments for measuring electrical quantities, IR46.

[7] International Electrotechnical Commission (IEC). (2003). Electricity metering equipment (a.c.) Particular requirements - Part 21: Static meters for active energy (classes 1 and 2). International Standard IEC 62053-21.

[8] Cataliotti, A., Cosentino, V., Lipari, A., Nuccio, S. (2009). Metrological characterization and operating principle identification of static meters for reactive energy: An experimental approach under nonsinusoidal test conditions. IEEE Transactions on Instrumentation and Measurement, 58 (5), 1427-1435.

[9] Georgakopoulos, D., Wright, P.S. (2007). Exercising the dynamic range of active power meters under nonsinusoidal conditions. IEEE Transactions on Instrumentation and Measurement, 56 (2), 369-372.
[10] IEEE. (2010). IEEE 1459-2010 IEEE Standard: Definitions for the measurement of electric power quantities under sinusoidal, nonsinusoidal, balanced, or unbalanced conditions.

[11] Cataliotti, A., Cosentino, V., Nuccio, S. (2008). A virtual instrument for the measurement of IEEE Std. 1459-2000 power quantities. IEEE Transactions on Instrumentation and Measurement, 57 (1), 85-94.

[12] Lu, Z.L., Li, M., Zhu, Z.Z., Zheng, J.Z., Wang, L., Eddy, S. (2012). Evaluation of the dynamic performance characteristic of electrical energy meters. In Conference on Precision Electromagnetic Measurements (CPEM), 1-6 July 2012, Washington, DC, USA.

[13] Huang, H.T., Lu, Z.L., Wang, L., Liu, L.J., Jia, Z.S., Huang, Q.L., Peng, X.J., Pan, Y. (2016). Dynamical waveforms and the dynamical source for electricity meter dynamical experiment. In Conference on Precision Electromagnetic Measurements (CPEM), 10-15 July 2016, Ottawa, Canada.

[14] Dix, C.H. (1982). Calculated performance of a digital sampling wattmeter using systematic sampling. IEE Proceedings A - Physical Science, Measurement and Instrumentation, Management and Education Reviews, 129 (3), 172-175.

[15] Dai, X.Z., Tang, T., Gretsch, R. (1993). Quasisynchronous sampling algorithm and its applications I. Principle and measurement of 'average' values of periodic signal. In IEEE Instrumentation and Measurement Technology Conference, 18-20 May 1993. IEEE, 88-93.

[16] Voloshko, A.V., Kotsar, O.I., Malik, O.P. (1995). An approach to the design of digital algorithms for measuring power consumption characteristic. IEEE Transactions on Instrumentation and Measurement, 10 (2), 607-612.

[17] Daniel, B., Dario, P. (2016). Accuracy analysis of the sine-wave parameters estimation by means of the windowed three-parameter sine-fit algorithm. Digital Signal Processing, 50, 12-23.

[18] Donoho, D. (2006). Compressed sensing. IEEE Transactions on Information Theory, 52 (4), 12891306. 
[19] Candes, E.J., Romberg, J., Tao, T. (2006). Robust uncertainty principles: Exact signal reconstruction from highly incomplete frequency information. IEEE Transactions on Information Theory, 52 (2), 489-509.

[20] Yang, J.B ., Liao, X.J., Yuan, X., Llull, P., Brady, D.J., Sapiro, G., Carin, L. (2015). Compressive sensing by learning a Gaussian mixture model from measurements. IEEE Transactions on Image Processing, 24 (1), 106-119.

[21] Zahedi, R., Krakow, L.W., Chong, E.K.P., Pezeshki, A. (2013). Adaptive compressive measurement design using approximate dynamic programming. In American Control Conference (ACC), 17-19 June, 2013, Washington, DC, USA, 2442-2447.

[22] Bertocco, M., Frigo, G., Narduzzi, C., Tramarin, F. (2014). Resolution enhancement by compressive sensing in power quality and phasor measurement. IEEE Transactions on Instrumentation and Measurement, 63 (10), 2358-2367.

[23] Du, Z.H., Chen, X.F., Zhang, H., Miao, H.H., Guo, Y.J., Yang, B.Y. (2016). Feature identification with compressive measurements for machine fault diagnosis. IEEE Transactions on Instrumentation and Measurement, 65 (5), 977-987.

[24] Yang, G., Tan, V.Y.F., Ho, C.K., Ting, S.H., Guan, Y.L. (2013). Wireless compressive sensing for energy harvesting sensor nodes. IEEE Transactions on Signal Processing, 61 (18), 4491-4505.

[25] Atia, G.K. (2015). Change detection with compressive measurements. IEEE Signal Processing Letters, 22 (2), 182-186.

[26] Mendelson, S., Pajor, A., Tomczak-Jaegermann, N. (2008). Uniform uncertainty principle for Bernoulli and subgaussian ensembles. Constructive Approximation, 28 (3), 277-289.

[27] Haupt, J., Bajwa, W., Raz, G., Nowak, R. (2010). Toeplitz compressed sensing matrices with applications to sparse channel estimation. IEEE Transactions on Information Theory, 56 (11), 58625875.

[28] Candès, E., Romberg, J. (2007). Sparsity and incoherence in compressive sampling. Inverse Problems, 23 (3), 969-985.

[29] Yan, W.J., Wang, Q., Shen, Y. (2014). ShrinkageBased alternating projection algorithm for efficient measurement matrix construction in compressive sensing. IEEE Transactions on Instrumentation and Measurement, 63 (5), 1073-1084.
[30] Yu, Y., Petropulu, A.P., Poor, H.V. (2011). Measurement matrix design for compressive sensingbased MIMO radar. IEEE Transactions on Signal Processing, 59 (11), 5338-5352.

[31] Li, G., Zhu, Z.H., Yang, D.H., Chang, L.P., Bai, H. (2013). On projection matrix optimization for compressive sensing systems. IEEE Transactions on Signal Processing, 61 (11), 2887-2898.

[32] Davenport, M., Boufounos, P., Wakin, M., Baraniuk, R. (2010). Signal processing with compressive measurements. IEEE Journal of Selected Topics in Signal Processing, 4 (2), 445-460.

[33] Park, J.Y., Wakin, M., Gilbert, A. (2014). Modal analysis with compressive measurements. IEEE Transactions on Signal Processing, 62 (7), 1655-1670.

[34] Agrež, D. (2010). Estimation and tracking of the power quality disturbances in the frequency domain. Measurement Science Review, 10 (6), 189-194.

[35] Alizadeh, M., Scaglione, A., Applebaum, A., Kesidis, G., Levitt, K. (2015). Reduced-Order load models for large populations of flexible appliances. IEEE Transactions on Power Systems, 30 (4), 1758-1774.

[36] Kabalci, E., Kabalci, Y. (2013). A measurement and power line communication system design for renewable smart grids. Measurement Science Review, 13 (5), 248-252.

[37] Duy, T.N. (2015). Modeling load uncertainty in distribution network monitoring. IEEE Transactions on Power Systems, 30 (5), 2321-2328.

[38] Petersen, H.M., Koch, R.G., Swart, P.H., Heerden, R.V. (1995). Modeling arc furnace flicker and investigating compensation techniques. In Industry Applications Conference, 8-12 October, 1995. IEEE, 1733-1740.

[39] Yang, S.B., Wu, M.L., Yao, X., Jiang, J.C. (2015). Load modeling and identification based on ant colony algorithms for $\mathrm{EV}$ charging stations. IEEE Transactions on Power Systems, 30 (4), 1997-2003.

[40] Zepernick, H.J., Finger, A. (2005). Pseudo Random Signal Processing: Theory and Application. Wiley.

Received January 25, 2018 Accepted September 24, 2018 